\title{
Kinematic and Kinetic Analysis of Rolling Motion in Normal Adults
}

\author{
Noboru SEKIYA ${ }^{1}$ and Masaaki TAKAHASHI ${ }^{1}$ \\ ${ }^{1}$ Department of Physical Therapy, School of Nursing and Rehabilitation Sciences, Showa University, Kanagawa 226-8555, \\ Japan
}

\begin{abstract}
The purpose of this study was to describe a rolling motion, which is common in normal adults and patients with motor disorder, and to try to understand the mechanism of the motion. Ten healthy young adults participated in the experiment and were requested to roll from supine to sidelying by pressing the floor with their contra-lateral foot. Their motions in the task were measured with a 3-D analysis system and a force platform. The hip abduction-adduction angle was at around the neutral position and was approximately constant throughout the rolling motion. The hip rotation angle was at a neutral or slightly internally rotated position at the beginning of the motion, and it externally rotated linearly toward the end of the rolling motion. These patterns were thought to be mechanically the most effective for the subjects to perform the motion as was also indicated by the kinetic analysis.
\end{abstract}

Key words: rolling, kinetic, kinematic, motor skill

(J Jpn Phys Ther Assoc 7: 1-6, 2004)

$\mathbf{R}$ olling is an important motor skill for our daily living. Physical therapy for patients with movement disorders sometimes includes the evaluation and treatment of rolling motion. To determine the quality and problems of rolling motion of patients, we must assess patients against some norm. We have, however, had no reliable norm for assessing the motion of patients. Some clinicians have proposed that specific rolling motion patterns are useful for treatment of patients with neurological dysfunction ${ }^{122)}$, but the validity of the rolling motions for treatment of motor disability is not clear ${ }^{3) 4}$. Furthermore, other rolling patterns might be appropriate for some handicapped people to acquire a movement skill.

Some developmental studies were performed to describe the change of rolling motion ${ }^{5) 6}$. These reports, however, were limited to descriptions of the rolling motions of infants and very young children. Richter et al. ${ }^{3 \text { ) }}$ categorized adult rolling movements into four patterns for each body region, head and trunk, upper extremities, and lower extremities, using videotape analysis. The results

Received: December 13, 2002

Accepted: March 29, 2003

Correspondence to: Noboru Sekiya, School of Nursing and Rehabilitation Sciences, Showa University, 1865 Tokaichiba-cho, Midori-ku, Yokohama, Kanagawa 226-8555, Japan.

e-mail: nsekiya@nr.showa-u.ac.jp showed great variability in the movement patterns used to roll from the supine to the prone position, in which 32 different combinations of movement patterns of the different body regions were exhibited. Nitta et al. ${ }^{7)}$ found three characteristic patterns in rolling motions of people with cerebral palsy using cluster analysis. However, they did not investigate the mechanism of the rolling motions. Taken together, these results suggest the need for examination of each rolling pattern or strategy separately in order to understand the motion.

While kinematic and kinetic analyses have been performed on various movement skills, e.g. walking ${ }^{4) 8-10)}$, running ${ }^{11) 12)}$, balance tasks ${ }^{13) 14}$, sit to stand ${ }^{15) 16)}$, to our knowledge none of the studies examined rolling motion kinematically and kinetically. Besides, the low relationship between the three body segments during rolling motion in the study of Richter et al. ${ }^{3)}$ might have been due to the low sensitivity of the measurement method (video analysis). This indicates that accurate kinematic and kinetic measurements are needed also in the research of rolling motion.

The purpose of this study was to describe a rolling motion, which is common in normal adults and patients with motor disorder, and to understand the mechanism of the motion kinematically and kinetically. 


\section{Methods}

Subjects

Ten healthy right-handed students ( 4 males and 6 females) without orthopaedic and neurological disorders participated in the experiment. Mean age was 21.9 $(\mathrm{SD}=2.7)$ years, mean height $162.7(\mathrm{SD}=7.1) \mathrm{cm}$, and mean weight $56.5(\mathrm{SD}=8.5) \mathrm{kg}$. All subjects gave their informed consent to undergo the experimental procedure.

\section{Experimental protocol}

The subjects lay supine on the floor covered with a carpet with their right foot on a force platform as shown in Fig. 1. The longitudinal axis of the trunk was parallel to the $\mathrm{X}$-axis. Their head, trunk and left lower extremity were in the neutral position and their arms were crossed in front of their chest. Their right legs were in the sagittal plane (markers c, d, and e) with their knees flexed at 90 degrees. They were requested to push the floor with their right foot and to roll over onto their left side with minimal movements of neck, trunk, and arms. This pattern of upper half of body was selected so that the function of lower half of body in the rolling motion could be clearly revealed. Seven subjects performed the task once and 3 subjects three times at their preferred speeds after several practice trials. For the latter subjects the first of the three trials were adopted in the kinematic analyses.

\section{Measurement system}

Fig. 1 shows the experimental set up. Kinematics of pelvis and right lower extremity was measured with a VICON370 system (Oxford Metrics) at $60 \mathrm{~Hz}$. Five infrared spherical reflexive markers were glued to relevant landmarks of the subjects (a-e, Fig. 1). Kinematic measurements were performed on every trial.

Floor reaction force to the right foot was measured by a force platform (KISTLER, 9287A). The kinetic data were collected at $60 \mathrm{~Hz}$, and synchronized with the kinematic data. Kinetic measurements were performed on three trials of the task for three subjects.

All data were filtered with a digital low path filter ${ }^{17)}$ at $6 \mathrm{~Hz}$.

\section{Definitions of experimental parameters (Fig 1)}

$\theta_{1}$, hip abduction, is the angle of segment cd from segment ab. $\theta_{2}$, hip rotation, is the angle of segment ed from segment $\mathrm{ab}$ in the transverse plane (Fig. 1B). $\alpha_{1}$ is the angle of the segment ab from the horizontal line on the transverse plane (Y-Z plane) (Fig. 1B). $\alpha_{2}$ is the angle of the vector of floor reaction force from the horizontal line on the transverse plane. Pelvic motion on the transverse plane during rolling movements was approximated to the total body rolling motion. $g$ is the instantaneous center of rotation (ICR) of the pelvis, and was defined as the intersection of 2 perpendicular bisectors: one was constructed by 2 consecutive points of left ASIS ( $a_{i}$ and $\left.a_{i+1}\right)$ and the other by those of right ASIS $\left(b_{i}\right.$ and $\left.b_{i+1}\right)$ (Fig. 1C). $\mathrm{f}$ is the center of pressure of the floor reaction force (Fig .1B). $\alpha_{3}$ is the angle between the force vector and the segment gf, and the angle of 90 degrees was thought to be most effective kinetically during rolling motion (Fig. 1B).

\section{Results}

Figure 2 shows movements of pelvis $\left(\alpha_{1}\right)$ on the transverse plane during rolling motions. Average maximum angle of rolling $\left(\alpha_{1}\right)$ and average movement time were 86.7 ( $\mathrm{SD}=9.9)$ degrees and $2.1(\mathrm{SD}=0.5) \mathrm{sec}$, respectively. Pelvises of the subjects rotated on the floor in a s-figure or gradually increasing function against time, suggesting that each rolling motion was similar and performed smoothly.

Figure 3 shows the relation between hip abductionadduction $\left(\theta_{1}\right)$ and pelvic rotation $\left(\alpha_{1}\right)$ in the transverse plane. The hip abduction-adduction was at around the neutral position (90 degrees) and was approximately constant throughout the rolling movements. However, every subject had a peak abduction angle in the mid-range of rolling motion. The mean peak abduction angle was 96.3 $(\mathrm{SD}=5.1)$ degrees with a rolling angle $\left(\alpha_{1}\right)$ of $45.4(\mathrm{SD}=7.0)$ degrees.

Figure 4 shows the hip rotation angle $\left(\theta_{2}\right)$ against pelvic rotation angle $\left(\alpha_{1}\right)$. The hip joint was at the neutral or slightly internally rotated position at the beginning of the rolling motions, and it externally rotated linearly toward the end of the rolling motion.

Figure 5 indicates the direction of floor reaction force $\left(\alpha_{2}\right)$ from the horizontal axis (Y-axis). It was slightly larger than 90 degrees at the beginning of rolling motion and gradually increased toward the end of the motion.

$\alpha_{3}$ is an approximate angle between "lever arm" of body rotation and floor reaction force to the right foot in the transverse plane. We supposed that the floor reaction force is most effective for the rolling when $\alpha_{3}$ is 90 degrees. While the angle was variable in the beginning and end of the movements as shown in Fig. 6, it was at around 90 degrees and roughly constant during the mid-range of rolling motion.

\section{Discussion}

Since rolling motion of normal subjects is so variable ${ }^{3)}$ or redundant, we thought that each movement pattern should be analyzed separately. In this research we studied a rolling motion, which was caused mainly by leg movement and is common to both normal and disabled subjects. In the rolling motions of this study, pelvises rotated in a s-figurefunction against time, indicating that they were smooth 


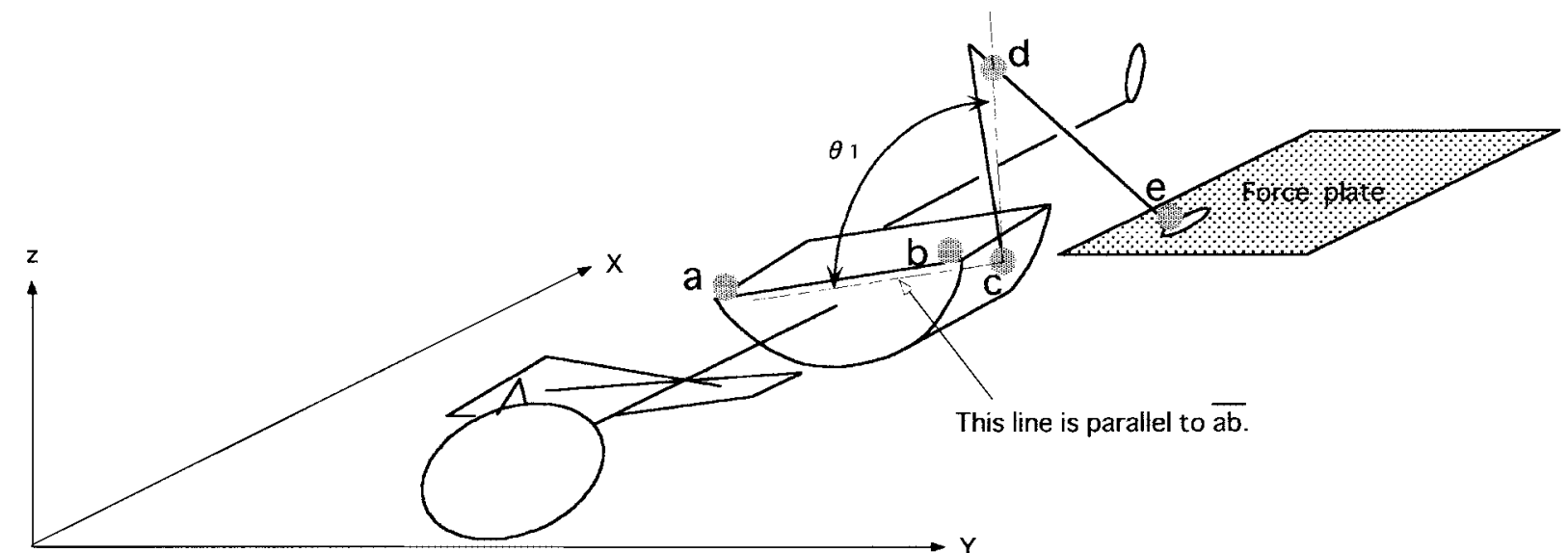

A. Spatial coodinate system and experimental settings.

Marker placement
a left ASIS
b right ASIS
c right greater trochanter
d right head of fibula
e right lateral malleolus

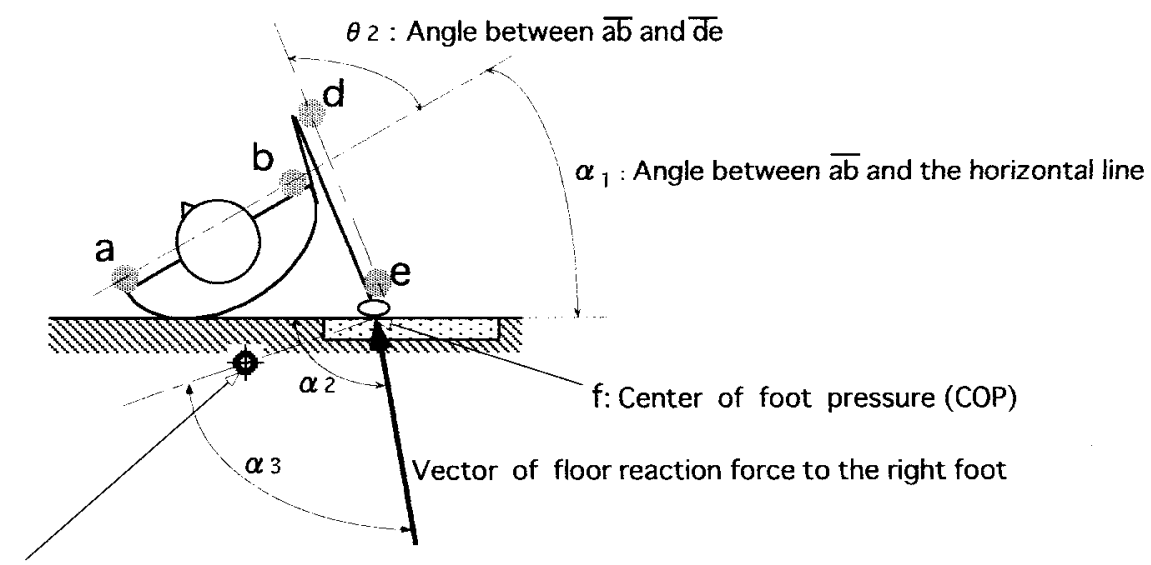

$\mathrm{g}:$ "Instantaneous center of rotation" (ICR) of pelvis

B. Parameters on the transverse plane ( $\mathrm{Y}-\mathrm{Z}$ plane).



C. Definition of instantaneous center of rotation(ICR).

$i+1$ is a time point $1 / 60 \mathrm{sec}$ after the time point $i$.

Fig. 1. Experimental settings and parameters. 


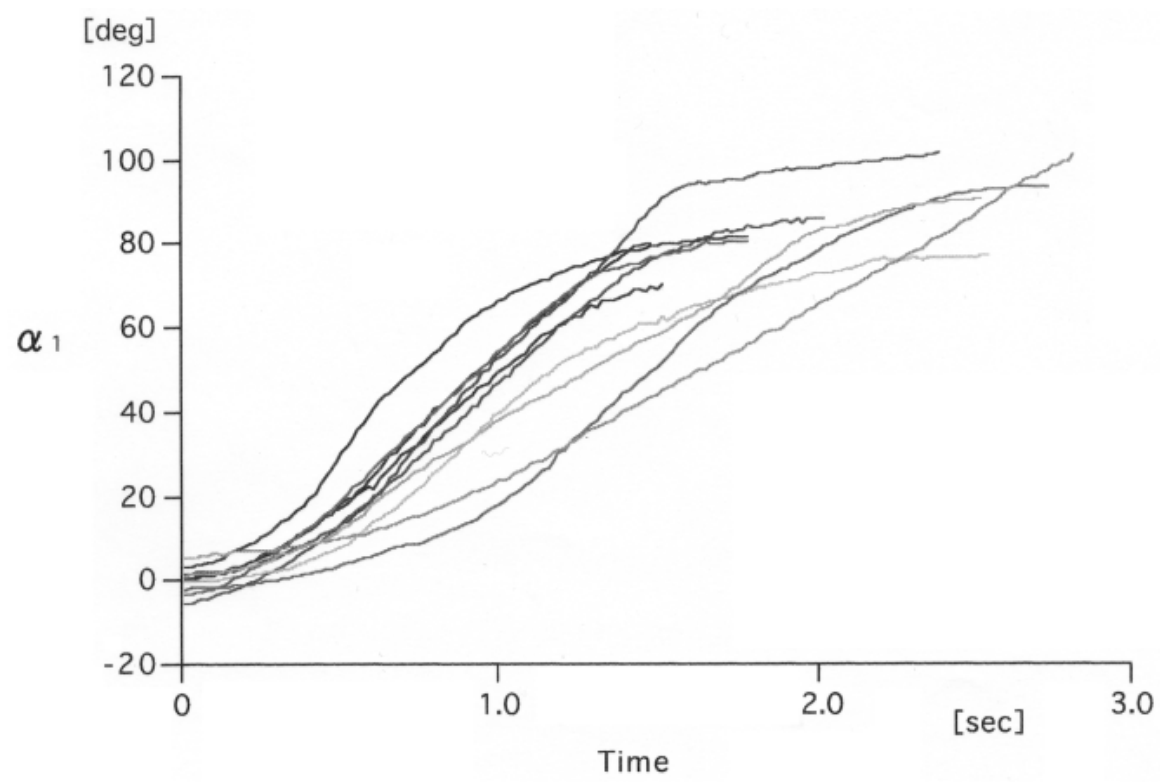

Fig. 2. Movements of pelvis on the transverse plane during rolling motions. Each line shows a trial for each subject.

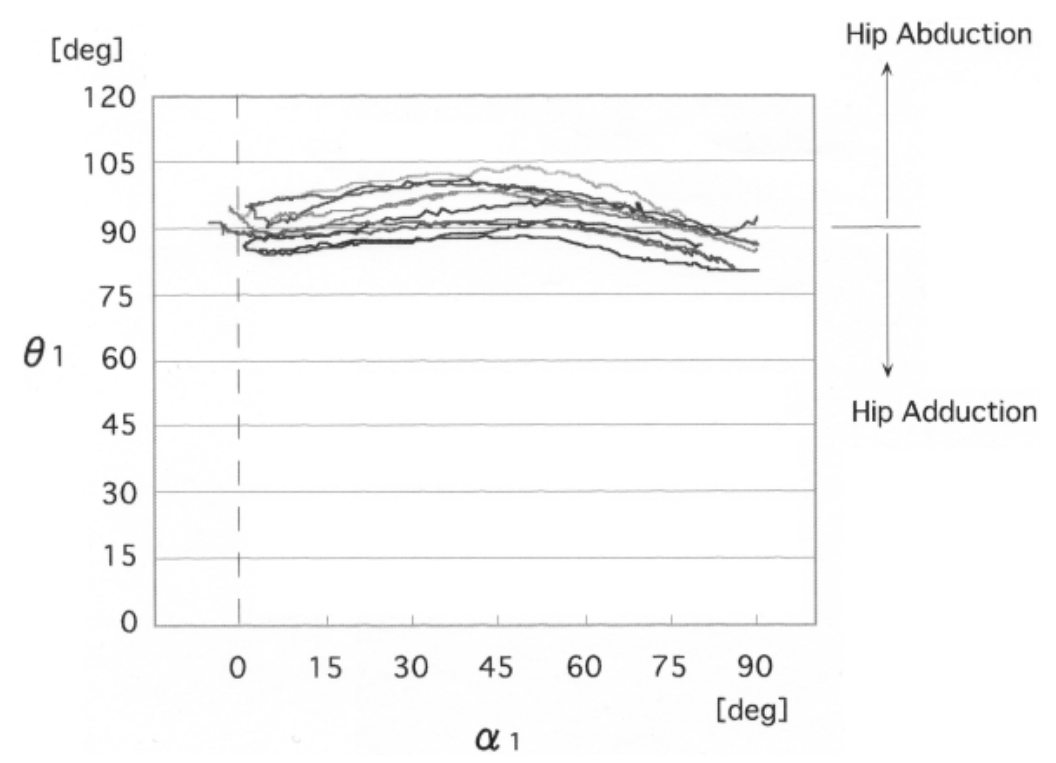

Fig. 3. Abduction-adduction $\left(\theta_{1}\right)$ of hip joints during rolling motions. $\alpha_{1}$ : Rotation of pelvis in the transverse plane.

movements. While the movement time was variable, the patterns of the movements were similar.

Hip abduction-adduction of the right hip joint was at around the neutral position throughout the rolling motions. This relation between pelvis and femur suggests that the neutral position of the hip joint in the sagittal plane is most effective for transmitting the floor reaction force to the pelvis and trunk. On closer examination, however, the hip joints abducted slightly in the first half of the rolling motions, and then reversed the direction. While this angle was rather small, the consistency of the pattern suggests that the motion is important for rolling.
The angle of the floor reaction force from the segment gf $\left(\alpha_{3}\right)$ was at around 90 degrees in the mid-range of rolling motion. This angle is dynamically most effective for rolling the body on the floor. External rotation of hip joint increased and the vector angle of floor reaction force to the right foot increased slightly with the rotation of pelvis. We think these produce the optimal relation between the force vector and the ICR. However, $\alpha_{3}$ at the beginning of rolling fluctuated extremely. Since the direction of floor reaction force $\left(\alpha_{2}\right)$ did not fluctuate, the fluctuation can be attributed to pelvic movements. Such movements of the pelvis are thought to be caused by irregular movements of the pelvis 


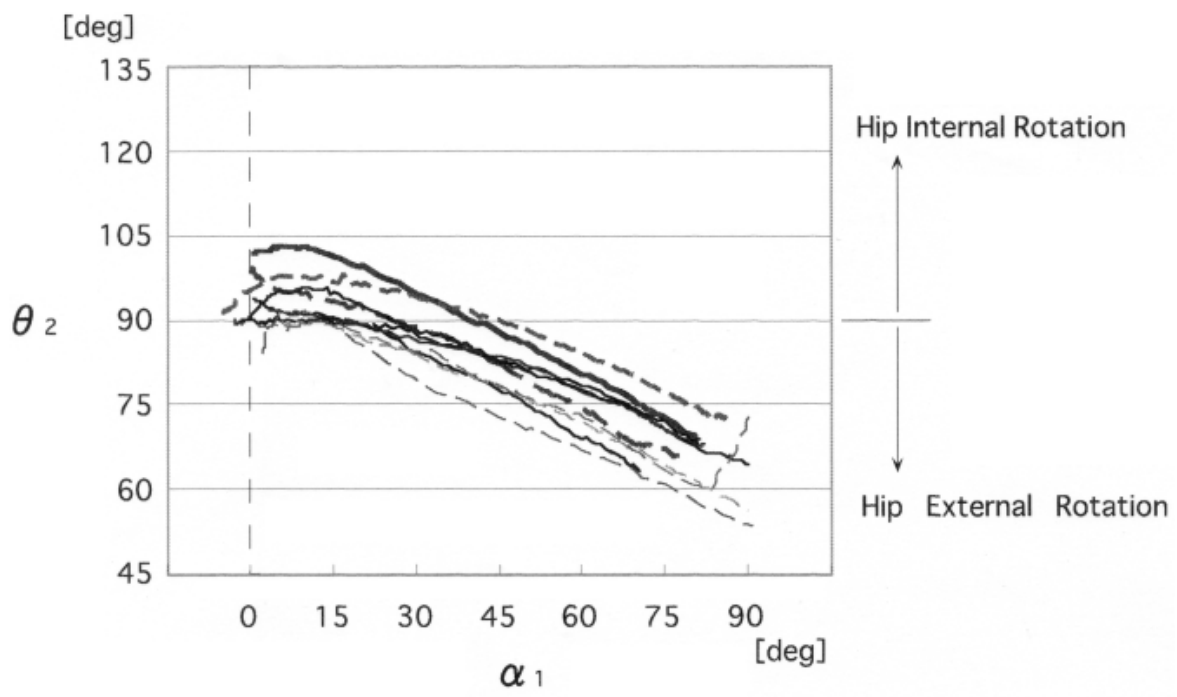

Fig. 4. Right hip rotation $\left(\theta_{2}\right)$ during rolling motions. $\alpha_{1}$ : Rotation of pelvis in the transverse plane.

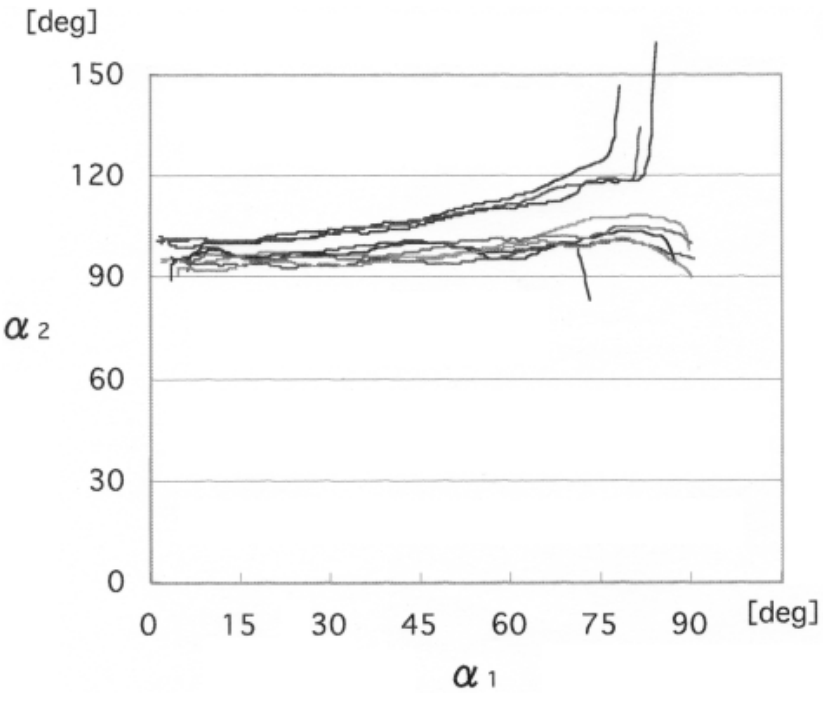

Fig. 5. Angle of floor reaction force to the right foot in the transverse plane. $\alpha_{1}$ : Rotation of pelvis in the transverse plane. $\alpha_{2}$ : Angle of floor reaction force from the horizontal line in the transverse plane.

and/or marker vibration other than rotational movements, because the human body is not sufficiently rigid.

To date, we have had no reliable standard or norm to evaluate rolling patterns of patients with motor disability. Some researchers have classified rolling motions of normal and handicapped persons, and interpreted the results only from developmental and/or neurological points of

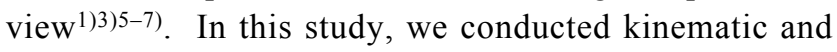
kinetic analysis of rolling motion and confined the analysis to a given rolling pattern, and revealed consistent relationships between pelvis and leg movements. Since the motion pattern exhibited here was also suggested to be mechanically optimal, it might be used as a norm or standard for assessment and therapy of patients with motor disability, especially with severe motor dysfunction. As the present study is preliminary and limited to only one pattern of rolling motion in normal young adults, however, further studies are needed to clarify the other patterns and detailed mechanisms of rolling motions. Moreover, unfolding the context in which each rolling motion emerges is another issue to be solved.

\section{References}

1) Bobath B: Adult Hemiplegia; Evaluation \& Treatment. 2nd ed. London, Heinemann Medical Books Ltd, 1978, pp 43-48.

2) Voss DE: Proprioceptive neuromuscular facilitation. Am J Phys Med 46: 838-898, 1967.

3) Richter RR: Description of adult rolling movements and hypothesis of developmental sequences. Phys Ther 69(1): 6371, 1989.

4) Shumway-Cook A, Woolacott MH: Motor Control: Theory and Practical Applications. 2nd ed, Baltimore, Lippincott Williams \& Wilkins; 2001, pp 351-352.

5) Gesell A, Amaturuda GS: Developmental diagnosis: Normal and Abnormal Child Development. 2nd ed, New York, Harper \& Row Publisher inc; 1947.

6) McGraw MB: Development of neuro-muscular mechanism as reflected in the crawling and creeping behavior of the human. The Journal of Genetic Psychology 58: 83-111, 1941.

7) Nitta O, Takaku N, Sasagawa Y, Yanagisawa K, Saito H: Cluster analysis on the Features of rolling over in cerebral palsy. The Journal of Tokyo Academy of Health Sciences 1(1): 103-106, 1998 (in Japanese).

8) Craik RL, Oatis CA(Editors): Gait Analysis. St. Missouri, Mosby, 1995.

9) Rose R, Gamble JG: Human Walking, 2nd ed, Baltimore, Williams \& Wilkins, 1994.

10) Winter DA: The Biomechanics and Motor Control of Human Gait: Normal, Elderly and Pathological, 2nd ed, Waterloo, Waterloo Biomechanics, 1991. 


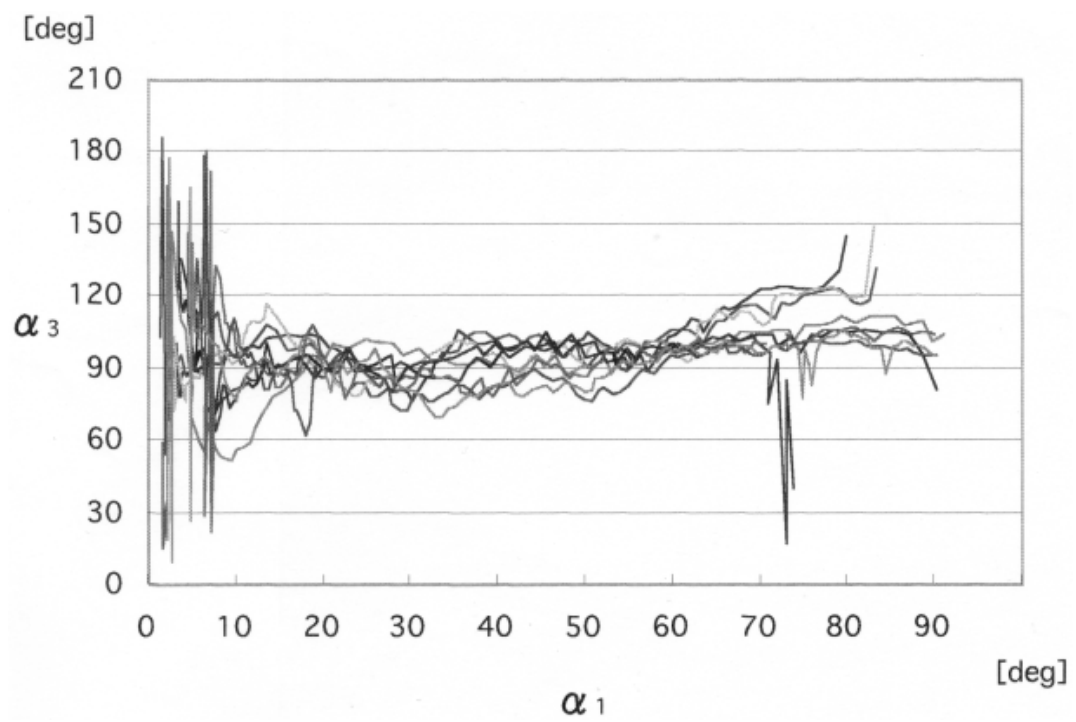

Fig. 6. Angle between "lever arm" of body rotation and floor reaction force $\left(\alpha_{3}\right)$ in the transverse plane. $\alpha_{1}$ : Rotation of pelvis in the transverse plane.

11) Dillman CJ: Kinematic analyses of running. In: Wilmore JH, Keogh JF. (eds.) Exercise and Sport Sciences Reviews. Vol. 3, New York, Academic Press, 1975, pp 193-218.

12) Mann RA: Biomechanics of running. In: D'Ambrosia RD (ed) Running Injury. SLACK, 1995.

13) Mouchnino L, Aurenty R, Massion J, Pedotti A: Coodination between equilibrium and head-trunk orientation during leg movement: a new strategy built up by training. Journal of Neurophysiology 67(6): 1587-1598, 1992.

14) Runge CF, Shupert CL, Horak FB, Zajak FE: Postural Strategies defined by joint torques. Gait and Posture 10: 161-
170, 1999.

15) Millington PJ, Myklebust BM, Shambes GM: Biomechanical analysis of the sit-to-stand motion in elderly persons. Arch Phys Med Rehabil 73: 609-617, 1992.

16) Yoshida $K$, Iwakura $H$, Inoue $F$ : Motion analysis in the movements of standing up from and sitting down on a chair. Scand J Rehab Med 15: 133-140, 1983.

17) Bryant JT, Wevers HW, Lowe PJ: Methods of data smoothing for instantaneous center of rotation measurements. Medical \& Biological Engineering \& Computer 22: 597-602, 1984. 